\title{
Climate predicts both visible and near-infrared reflectance in butterflies
}

\author{
Changku Kang ${ }^{1}$, Sehyeok $\mathrm{Im}^{2}$, Won Young Lee ${ }^{2}$, Yunji Choi ${ }^{3}$, Devi Stuart-Fox ${ }^{4}$, and \\ Blanca Huertas ${ }^{5}$
}

${ }^{1}$ Mokpo National University

${ }^{2}$ Korea Polar Research Institute

${ }^{3}$ Imperial College London

${ }^{4}$ University of Melbourne School of BioSciences

${ }^{5}$ Natural History Museum London

February 1, 2021

\begin{abstract}
Climatic gradients frequently predict large-scale ecogeographical patterns in animal coloration, but the underlying causes are often difficult to disentangle. We examined ecogeographical patterns of reflectance among 343 European butterfly species and isolated the role of selection for thermal benefits by comparing visible and near-infrared (NIR) wavebands. NIR light accounts for $\sim 50 \%$ of solar energy but cannot be seen by animals so functions primarily in thermal control. We found that reflectance of both dorsal and ventral surfaces shows thermally adaptive correlations with climate. This adaptive variation was more prominent in NIR than visible wavebands and for body regions (thorax-abdomen and basal wings) that are pivotal for thermoregulation. Thermal environments also predicted the reflectance difference between dorsal and ventral surfaces, which may be due to modulation between requirements for heating and cooling. These results highlight the importance of climatic gradients in shaping the reflectance properties of butterflies at a continent-wide scale.
\end{abstract}

\section{Introduction}

Large-scale ecogeographical gradients can explain variation in diverse traits, from body size (Ashton 2002) to colour (Friedman \& Remeš 2017). Several ecogeographical patterns have been formalised into rules; yet there is persistent debate regarding underlying causes and the taxa to which they apply (Gaston et al. 2008; Chown \& Gaston 2010). This problem is epitomised by Gloger's rule and Bogert's rule, which both describe ecogeographical patterns of melanisation. Gloger's rule describes the tendency for heavily pigmented (darker) forms to be found in hotter and more humid regions (Delhey 2017, 2019). This relationship may be driven by one or more factors including camouflage in low light environments (Zink \& Remsen Jr 1986; Cheng et al. 2018), protection from ultraviolet light or parasites (Burtt Jr \& Ichida 2004; Chaplin 2004), or pleiotropic effects of genes regulating both climatic adaptations and melanin-based coloration (Ducrest et al. 2008). Bogert's rule (also termed the thermal melanin hypothesis) describes the tendency for darker animals to occur in colder regions because darker colours absorb more solar radiation, thus providing thermal benefits (Bogert 1949). Traditionally, Bogert's rule has been applied to ectotherms and Gloger's rule to endotherms; however, the accumulated evidence suggests that both rules may apply broadly to ectotherms or endotherms (Trullas et al. 2007; Delhey 2018; Galván et al. 2018; Delhey et al. 2019). Efforts to reconcile the seemingly opposing effects of these rules have so far been hampered by the difficulty of disentangling the underlying drivers.

Most of the evidence that supports either Gloger's or Bogert's rule relates to visible colour (all or part of the 
wavelengths range from 300-700 nm); however, the spectrum of direct sunlight extends well beyond this range. Wavelengths from 700-1400 nm (near-infrared, NIR) include approximately $50 \%$ of solar energy (Stuart-Fox et al.2017) and can therefore strongly affect heat gain. By contrast, NIR does not directly affect camouflage because little or no NIR light can be seen by animals (Stuart-Fox et al. 2017). Examining ecogeographical gradients in NIR reflectance can therefore help to distinguish underlying drivers of ecogeographical patterns of animal coloration (Cuthill et al. 2017; Stuart-Fox et al. 2017; Ruxton et al. 2018). To date, ecogeographical patterns of NIR reflectance have only been examined in Australian birds and butterflies (Medina et al. 2018; Munro et al. 2019), which both show thermally adaptive variation. However, Australia is a hot and dry continent so it remains unclear whether climatic gradients in NIR reflectance exist for other taxa or climates.

Butterflies are a model group to investigate ecogeographical patterns of light manipulation due to their thermal biology and extraordinary diversity in coloration. They are primarily ectothermic like many insects and regulate their body temperature through both physical and behavioural traits (Clench 1966). Physical properties of the thorax and basal wings (i.e. parts of the wings that are close to thorax) directly affect the temperature of flight muscles through heat conduction (Heinrich 1974). The wings beyond the basal region may have less impact on thermoregulation because there is less haemolymph circulation and fewer vascular extensions that can carry significant quantities of heat to the thorax (Arnold 1964; Kammer \& Bracchi 1973; Wasserthal 1983; Kingsolver 1987). However, wings can overheat quickly under direct sunlight due to their low thermal capacity, and butterflies have evolved sophisticated wing scale structures to control wing temperature through radiative cooling (Tsai et al. 2020). Butterflies also regulate their temperature through various behavioural mechanisms including dorsal and lateral basking (opening/folding wings to expose thorax and wing surface), ground-contact, orientating themselves in relation to the position of the sun, and shivering (Clench 1966). The reflectance of butterflies plays a crucial role during behavioural thermoregulation such as dorsal (wings open) and lateral (wings closed) basking because the efficacy of these behaviours depends on how much light their body and wings absorb (Kingsolver 1987, 1988). The reflectance of both (especially dorsal) regions seems to play a key role in warming up during basking while those of ventral regions seem to be additionally related to preventing overheating (Kingsolver 1987). Thus, thermal pressures may act differently on dorsal and ventral surfaces which consequently result in the evolution of reflectance differences between dorsal and ventral surfaces. Specifically, higher dorsal-ventral contrast is predicted to evolve in species that are found in heat-stress environments where the benefit for high ventral reflectance for cooling is greater. However, this prediction has yet to be formally tested.

In this study, we tested whether climate predicts the reflectance of both dorsal and ventral regions of 343 European butterfly species using full-spectrum photography of museum specimens. We compiled climatic niche characteristics of each species and tested multiple hypotheses regarding ecogeographical patterns of butterfly reflectance after controlling for phylogeny and phylogenetic uncertainty (Schweigeret al. 2014). We specifically addressed four questions regarding ecogeographical variation in butterfly reflectance: (1) does butterfly reflectance follow the patterns predicted by Bogert's rule in both visible and near-infrared wavebands? (2) does NIR reflectance show thermally adaptive patterns independent of visible reflectance? (3) does butterfly reflectance follow the patterns predicted by Gloger's rule? (4) does climate predict ventraldorsal contrast in butterflies?

\section{Methods}

\section{Photography}

We photographed 684 butterfly specimens from 343 species held in the Lepidoptera collections at the Natural History Museum in London (NHMUK). This includes species from all six families, covering approximately $70 \%$ of all species found in Europe. The distribution of analysed species ranged from $34^{\circ}$ to $70^{\circ} \mathrm{N}$ and $10^{\circ}$ $\mathrm{W}$ to $44^{\circ} \mathrm{E}$. The mean temperature over a species' distribution varied from -2.7 to $18{ }^{\circ} \mathrm{C}$, and the mean precipitation varied from 353 to $1544 \mathrm{~mm}$ per year. For each species, we selected and photographed two specimens whenever available and chose specimens with well-preserved wings and body. For the polymorphic species, we photographed each morph once. We focused on capturing inter- rather than intra-specific variation because we were primarily interested in evolutionary factors that have shaped broad-scale interspecific 
ecogeographic patterns. We note that most specimens were collected before 1980; however a previous study found no effect of specimen age on visible and near-infrared reflectance in butterflies (Munro et al. 2019) and any potential degradation will contribute variation within species but is unlikely to mask interspecific variation or affect biological conclusions at the scale of our analysis.

Photography was done in a dim room using two light bulbs simultaneously: an LED bulb (True-light LED 12W E27, Frankfurt, Germany CRI index 98, spectral power distribution for this bulb is provided in Fig. S1) and a 3,000K tungsten-halogen lamp (150W, Long Life Lamp Company, Harrow, UK). The tungstenhalogen lamp emitted all UV, visible, and NIR light. We set up these two bulbs approximately $60 \mathrm{~cm}$ above the photographic spot. A full-spectrum converted DSLR camera (Nikon D7000 converted by Lifepixel, Mukilteo, WA, USA; maximum) was set directly above the photographic area. We positioned the camera slightly below the bulbs to avoid light reaching directly to the camera lens (Jenoptik UV-VIS-IR $60 \mathrm{~mm}$ 1:4 APO Macro lens; transmission waveband is between 290 to $1500 \mathrm{~nm}$ ). We used lens filters (Baader, Mammendorf, Germany) to capture ultraviolet (U-Venus filter), visible (UV/IR cut filter), and near-infrared wavelengths ranges (IR-Pass filter). These filters completely blocked wavelengths outside the transmission range and enabled us to photograph each specimen in three spectral ranges: ultraviolet $(320-380 \mathrm{~nm})$, visible $(400-680 \mathrm{~nm})$, and near-infrared $(670-1050 \mathrm{~nm} ; 1050 \mathrm{~nm}$ is the maximum sensitivity of the camera sensors provided by the manufacturer) ranges, respectively. Our analysed spectral range $(320-1050 \mathrm{~nm})$ captures approximately $80 \%$ of the energy in solar irradiation. We changed lens filters with a minimum disturbance to the camera body using a combination of magnetic lens adapters and filter holders (Manfrotto, Cassola, Italy).

To fix each specimen, we used a square paper box floored with styrofoam $(17 \times 10 \times 5 \mathrm{~cm})$. We placed each specimen at the centre with a $99 \%$ reflectance standard (WS-1-SL, Labsphere, NH, USA) on the upper-right corner of the box. Camera settings were constant (ISO 400, F8) except for the shutter speed which varied depending on the type of filters (1.3 s for ultraviolet, $1 / 500 \mathrm{~s}$ for visible, $1 / 800 \mathrm{~s}$ for near-infrared photos). This produced 4,104 images saved in raw format (see Fig. S2 for sample images).

\section{Image analysis}

To remove non-linearity of the camera response, we converted all raw photos to linearised TIFF format images using Dcraw (Coffin 2008). Then we rescaled each colour channel to ensure that the $99 \%$ reflectance standard had the corresponding value. After this process, RGB values of each pixel (ranging from $0-255$ for each colour channel) scale linearly with reflectance of $0-100 \%$. We used the B channel for ultraviolet

photos, R, G, and B channels for visible photos, and R and B channels for near-infrared photos because the camera sensors have sensitivity in the corresponding spectral range.

For all images, we measured mean channel values of three body regions: thorax-abdomen (both of which are important for thermoregulation due to haemolymph circulation between them (Rawlins 1980); we refer to thorax for brevity), basal wings, and entire wings. Thus, we measured six different body regions for each specimen: dorsal thorax (DT), dorsal basal wings (DB), dorsal entire wings (DE), ventral thorax (VT), ventral basal wings (VB), and ventral entire wings (VE). Because butterfly wings were symmetrical, we measured wing regions only from one side (left). For each region, we averaged the pixel values of all measured channels to get a mean reflectance value. Then we calculated the mean reflectance of each body part over 1) VIS (320 - $700 \mathrm{~nm}$; animal-visible range), 2) NIR (700 - $1050 \mathrm{~nm}$; animal-invisible near-infrared range), and 3) VIS-NIR $(320-1050 \mathrm{~nm})$ ranges. For example, VIS reflectance was calculated by $(60 u+360 v) /(60+$ 360 ), where $u$ and $v$ are the mean reflectance of ultraviolet and visible-range images respectively, and the numbers 60 and 360 are wavebands of the spectral images. We measured the entire wing area for each species (averaged among specimens) and used this as a size index for each species.

\section{Climatic variables}

We used nine climatic niche variables for each species in our analysis. We obtained the mean, minimum, and maximum annual temperature $\left({ }^{\circ} \mathrm{C}\right)$, and annual precipitation sum $(\mathrm{mm})$ from an open dataset CLIMBER (Climatic niche characteristics of the butterflies in Europe) and averaged each variable across each species' 
distribution (data from 1971 to 2000) (Schweiger et al. 2014). The other five climatic variables, namely solar irradiation $\left(\mathrm{kJ} \mathrm{m}^{-2} \mathrm{day}^{-1}\right)$, isothermality, temperature seasonality, precipitation seasonality, and water vapour pressure $(\mathrm{kPa})$ were compiled for every $50 \mathrm{~km}$ using WorldClim data (Fick \& Hijmans 2017) (data from 1970 to 2000) and averaged over all years and across species' range.

\section{Data analysis}

We used R 4.0.2. for data analysis (R Core Team 2017). First, we reduced the dimensionality of the climatic variables using principal component analysis (PCA). Then we performed a phylogenetically controlled analysis to examine the relationship between size, climatic factors (PC1 and PC2; see results), and the mean butterfly reflectance over VIS-NIR range. The phylogenetic relationships of the analysed species were inferred from a published time-calibrated molecular phylogenetic tree (Wiemers et al. 2020). To account for the uncertainties in topology and node age, we generated 1,000 trees randomly sampled from the posterior distribution and performed the analysis using all 1000 trees.

For each tree, we fitted multivariate phylogenetic regressions implemented in the 'mvMORPH' package (Clavel et al. 2015). This enabled us to include multiple response variables in a single model. We set size, climatic principal components (PCs; up to PC2), and the interaction between size and climatic PCs as predictor variables. Our response variables consisted of the mean reflectance of the six body regions (DT, $\mathrm{DB}, \mathrm{DE}, \mathrm{VT}, \mathrm{VB}, \mathrm{DE})$. We compared the goodness of fit of the models assuming either Brownian motion, Ornstein-Uhlenbeck, Early Burst, or Pagel's $\lambda$ models of trait evolution and used the models with the lowest Generalized Information Criterion (GIC) (Konishi \& Kitagawa 1996; Hernández et al. 2013). OrnsteinUhlenbeck models showed slightly better fit than other models in multivariate phylogenetic regressions, but Pagel's $\lambda$ models outperformed others in allpost-hoc PGLS models. Thus, we consistently assumed Pagel's $\lambda$ models of trait evolution. Nevertheless, the results were robust regardless of which models we assumed. We also used GIC to select the best model among all candidate models.

Using the predictors that remained significant in the above model, we performed post-hoc phylogenetic generalised least squares (PGLS) on the mean reflectance of each body region to further examine which body regions were specifically explained by each predictor. We implemented a 'gls' function to run PGLS (Revell 2012). We iteratively performed PGLS on 1,000 trees for all body regions and estimated the 95\% confidence intervals of statistics. The $95 \%$ confidence limits of statistics from all analyses using 1000 trees varied only slightly and showed essentially the same results as the MCC tree results (all parameters estimated were within \pm 0.02 range) because of low phylogenetic uncertainty. Thus, we report the results from the MCC tree only in the results. Here, we used Akaike Information Criteria (AIC) to select the best model and controlled for the false-discovery rate by adjusting P-values (Benjamini \& Hochberg 1995). The strength of phylogenetic signal $\lambda$ was estimated using the maximum clade credibility (MCC) tree.

We additionally examined the effects of our predictors on VIS reflectance and NIR reflectance separately using the same modelling approach and model structure. To examine whether NIR reflectance showed adaptive features even after accounting for its high correlation with VIS reflectance, we fitted linear regressions to each NIR reflectance (for each body region) using log form of VIS reflectance as a predictor (average $r^{2}$ among all body regions [?] 0.77) and extracted residuals of the fitted models. These residuals indicate the degree of NIR reflectance independent of VIS reflectance (i.e. after accounting for the correlation between VIS and NIR reflectance). Though the use of residuals has been criticised due to potential for biased parameter estimation (Freckleton 2009), residuals are appropriate when the effect of one predictor on a response variable should be controlled 'before and independent' of the other predictors such as in our case; the effects of VIS reflectance on NIR reflectance should be considered before estimating the effects of other climatic and size variables and should not affect the parameters of other predictors in the model (see Supplementary materials for the statistical justification of using residuals). We fitted multivariate phylogenetic regressions using size, climatic PCs, and the interaction between those two as predictors and the residuals for each body region as response variables. Then we performed a post-hoc PGLS analysis for each body region using the retained significant terms as predictors as above. 
In the main results, we analysed all specimen images regardless of the sex and the presence of sexual dimorphism. However, because sexually dimorphic male reflectance is likely to be heavily shaped by sexual selection (Lande 1980; van der Bijl et al. 2020), we repeated the analysis without sexually dimorphic male specimens and compared the results.

For the analysis of ventral-dorsal reflectance differences, we first compared the reflectance between dorsal and ventral parts using paired t-tests. Next, to examine whether climate variables predict these differences, we calculated the reflectance differences between ventral and dorsal parts by subtracting dorsal reflectance from ventral reflectance for each body region. Using these ventral-dorsal differences in the three body regions as independent variables, we fitted phylogenetic multivariate multiple regressions with climatic PCs, size, and the interaction between them as dependent variables. We then performed post-hoc PGLS analyses for each body region using the retained significant terms as predictors.

\section{Results}

The overall relationship between climatic variables and VIS-NIR reflectance

Climate variables were the first two principal components (PCs) from a Principal Components Analysis (PCA) of 9 climatic niche variables. Climate PC1 was higher in species that inhabit in warmer, drier climatic conditions and correlated most strongly with mean temperature $(r=0.98)$, solar radiation $(r=$ 0.88 ) and water vapour pressure $(r=0.87)$ (Fig. 1 left panel). Climate PC2 was higher in species that inhabit areas with higher mean precipitation $(r=0.78)$ and low precipitation seasonality $(r=-0.77)$ (see Tables S1,2 for the full PCA results).

Climate was a significant predictor of butterfly reflectance for all body regions. In the multivariate phylogenetic regression, mean total VIS-NIR reflectance was predicted by $\mathrm{PC} 1\left(F_{6,334}=12.58, P<0.001\right)$, PC2 $\left(F_{6,334}=3.66, P=0.002\right)$, and size $\left(F_{6,334}=7.44, P<0.001\right)$ (other variables excluded from the model with $P>0.2)$. Phylogenetic signal was high $(\lambda=0.76)$. Post-hoc PGLS models that used the mean reflectance of each body region as a response variable showed that for all body regions, species from colder environments (lower PC1) had lower reflectance, which corresponded to a gradual decrease in the reflectance of butterfly assemblages across latitudinal gradients (Table 1; Fig. 2,3). PC2 was also significant for all body regions (except for the ventral thorax), with species from high precipitation environments having lower reflectance than species from drier environments. Size predicted the reflectance of both dorsal and ventral entire wing: smaller species tended to show higher mean entire wing reflectance than larger species. No other variables remained significant. The results from the models excluding sexually dimorphic males showed the same trends except that PC2 became non-significant for all ventral regions (Supplementary materials, Tables S3,4). Phylogenetic signals were consistently high for all body regions (all $\lambda>0.72$ ).

The relationship between overall (VIS-NIR) reflectance and climate was evident for both VIS and NIR wavelength ranges but driven more by NIR reflectance for the thorax and basal wing. Results for VIS and NIR reflectance separately were essentially consistent with the overall reflectance models (see Supplementary materials, Tables S5,6, Figs. S3,4 for the full results). PC1 predicted VIS and NIR reflectance for all body regions; however, the relationship was more robust (larger coefficients) for NIR reflectance for the thorax and basal wing, but not the entire wing (dorsal and ventral). PC2 also predicted the reflectance of most body regions except for ventral thorax and basal regions in the VIS range, and the ventral thorax in the NIR range. Size did not predict VIS reflectance of butterflies but predicted NIR reflectance of the entire wing (both dorsal and ventral); smaller butterflies showed higher NIR reflectance than larger butterflies. The direction of this relationship (i.e. whether coefficients had negative or positive values) was always the same as the overall reflectance models for all significant predictors.

We additionally examined whether NIR reflectance show adaptive variation after accounting for its high correlation with VIS reflectance (see methods). Analyses using residuals from the linear regression between NIR and $\log$ (VIS) as response variables confirmed the importance of NIR reflectance of the thorax and basal wing in the overall correlations between reflectance and climate. The multivariate phylogenetic regression model showed that $\mathrm{PC} 1\left(F_{6,334}=4.63, P<0.001\right), \mathrm{PC} 2\left(F_{6,334}\right.$ range $=2.34, P=0.03$, and size $\left(F_{6,334}\right.$ 
$=7.69, P<0.001$ ) were significant predictors of NIR residuals (Table 1). Post-hoc PGLS revealed that the residuals of ventral thorax and basal wing regions were higher in species from hotter environments (with higher PC1; Fig. 4). PC2 predicted dorsal thorax, basal wing, and ventral basal wing regions. Size predicted dorsal and ventral entire wing regions. The trends of the significant relationships were the same as the overall reflectance models (Table S7). All other variables were non-significant with $P_{\text {adj }}>0.1$.

\section{The relationship between climatic variables and ventral-dorsal differences}

Here, we tested whether climate predicts dorsal-ventral reflectance contrast in butterflies. Ventral reflectance showed higher reflectance than dorsal reflectance for all three body regions in most of the species (all $t_{342}>$ $25, P<0.001$, Fig. S5). Phylogenetic signal was present in these differences $(\lambda=0.66)$. In the best model, only $\mathrm{PC} 1\left(F_{6,334}=17.01, P<0.001\right)$ was a significant predictor (other terms removed with $\left.P>0.2\right)$ with species from colder environments showing a greater difference between dorsal and ventral reflectance.Posthoc PGLS models revealed that this relationship was present for both the thorax (coef $=1.47, t=2.63, P_{\text {adj }}$ $=0.01)$ and basal wing regions $\left(\right.$ coef $\left.=1.14, t=4.92, P_{\text {adj }}<0.001\right)$, but not in entire wing region $($ coef $=$ $\left.0.00, t=-1.42, P_{a d j}=0.16\right)$. This indicates that butterflies in warmer environments evolved higher contrast between ventral and dorsal reflectance in body regions that are important for thermoregulation.

\section{Discussion}

The reflectance of European butterflies followed the ecogeographical patterns predicted by Bogert's rule: butterfly species in colder regions showed lower reflectance than species in warmer regions in both VIS and NIR wavebands. This pattern was consistent for dorsal and ventral reflectance of all body regions. The consistent pattern for both VIS and NIR wavebands is not surprising because reflectance in these two parts of the spectrum was highly correlated. However, even after removing the effect of this correlation, residual NIR reflectance of the ventral thorax-abdomen and basal wings still showed thermally adaptive patterns. Thus, our results clearly demonstrate that thermal benefits drive ecogeographical patterns of reflectance in European butterflies.

We also found evidence for Gloger's rule in European butterflies. Delhey proposed two different definitions of Gloger's rule: a simple version states that animals are darker in a more humid environments while a more complex version includes differential effects of humidity and temperature on different types of melanin pigments (Delhey 2019). Our results generally follow the patterns predicted by the simple version of Gloger's rule: after accounting for the effect of temperature-related variables (PC1), reflectance of most body regions was lower in species found in a more humid area (i.e. with higher mean precipitation). The trends were consistent for all body regions (coefficients of PC2 in Tables 1 and S5-6 are all negative; although this relationship was not statistically significant for the ventral thorax). This suggests that not only thermal environments but the degree of humidity also affects the ecogeographical patterns of butterfly reflectance. Camouflage in warm and humid environments could drive this relationship because such environments are usually covered by darker vegetations and under low light conditions, favouring the occurrence of darker species (Xinget al. 2016; Cheng et al. 2018).

In accordance with a previous study on Australian butterflies (Munroet al. 2019), we found similar high correlations between VIS and NIR reflectance. This is not surprising because reflectance varies continuously and often gradually across the spectrum and the degree of VIS reflectance generated by pigments, such as melanins, often correlate with their NIR reflectance (Alla et al. 2009). However, structural colour, which is common in butterflies, can produce a wide diversity of spectral shapes with multiple peaks in different parts of the spectrum, potentially enabling VIS and NIR properties to respond differently to selection. Our results suggest that selection for thermal benefits has shaped both VIS and NIR reflectance in European butterflies because both showed patterns consistent with Gloger's rule. However, the ventral basal wing and thorax regions also showed thermally-adaptive variation independent of their VIS reflectance. This implies that butterfly reflectance might be tuned to modulate signalling or camouflage needs in VIS reflectance and thermoregulatory needs in NIR reflectance despite the constraints imposed by the correlations between them (Munro et al. 2019). 
Though entire wing reflectance also showed thermally adaptive ecogeographical patterns, the strength of this relationship was weaker than for the thorax and basal wing regions. The evolution of butterfly reflectance is likely to be affected by multiple competing functions, including camouflage and signalling (Silberglied 1984; Kapan 2001; Chenget al. 2018; van der Bijl et al. 2020). The stronger climate-reflectance relationships for the thorax and basal wing area suggest that the relative importance of thermoregulation is greater for these body regions, consistent with their more critical role in thermoregulation (Wasserthal 1983). Basal wing and thorax regions comprise a smaller area than the entire wing and are pivotal for thermoregulation due to haemolymph circulation and proximity to flight muscles (Arnold 1964), thus they may be less affected by competing selective pressures other than selection for thermal benefits.

Our results show that larger species have lower entire wing reflectance than smaller species in the NIR but not VIS wavebands. In other words, size correlates with NIR reflectance, but not colour. Why have larger butterflies evolved lower NIR reflectance of the wings independent of both climate and colour? Cryptic NIR adaptations of wings could contribute to thermoregulation. Although heat transfer from the wings to the thorax through conduction may be limited, heat transfer may be greater for larger than smaller wings. Larger butterflies have been shown to perform better at controlling temperature and affording elevated body temperature (Gilchrist 1990; Bladon et al. 2020). Alternatively, the wings may function as solar concentrators and reflect the solar energy radiated across the entire wings towards the thorax (Shanks et al. 2015). This effect should be greater for larger species because larger wings can reflect more solar radiation towards the thorax than smaller ones. Thus, larger species may have evolved lower NIR reflectance than smaller species to modulate the amount of reflective energy from the wings to the thorax. A third possibility is that larger butterflies may prefer to be active in the shade and crepuscular hours which could also drive the evolution of lower NIR reflectance (Xing et al. 2016). The underlying reason for the observed size-reflectance relationship remains to be tested.

Butterflies use both dorsal and ventral basking, and both dorsal and ventral reflectance can contribute to the process of heat transfer, depending on basking behaviour (Clench 1966; Kingsolver 1985). However, ventral regions are additionally exposed during cooling down when butterflies close their wings tightly to minimise the absorption of solar radiation (Clench 1966). To avoid the absorption of unnecessary heat during cooling down, it may be equally important to have high ventral reflectance, especially for species in warmer climates. Thus, the reflectance of ventral regions in butterflies may be a result of evolutionary modulation between two conflicting selective pressures: absorbing light energy when heating up and reflecting it when cooling down. In cold climates, selection for low reflectance to enable rapid warming may prevail, while in hot climates, there may be stronger selection for high ventral reflectance to facilitate cooling. Indeed, our results demonstrate that ventral surfaces had higher reflectance than dorsal surfaces in most species and the difference was larger in warmer climates. Notably this relationship was only present for the thorax and basal wing regions that are crucial for thermoregulation. This suggests that the evolution of the ventral surfaces of butterflies is affected by thermoregulatory pressures related to both heating and cooling.

Thermal benefits have been considered as one of the major selective agents that operate on butterfly reflectance (Kingsolver 1988; Hegnaet al. 2013). Our findings provide the most comprehensive evidence to date that climatic gradients have shaped both visible and near-infrared reflectance of butterflies consistent with both Gloger's and Bogert's rules. We also show that not all body regions were equally affected, but the observed climate-reflectance relationship was stronger for body regions that play a greater role in thermoregulation. This highlights that the relative strength of competing selective pressures (e.g. signalling, camouflage, heating up, or cooling down) may vary between different body parts and these collectively have affected the evolution of the reflectance properties of butterflies.

\section{Acknowledgements}

We are grateful to Natural History of Museum London for allowing us to photograph their invaluable specimens. We thank to Alessandro Giusti for his help with the collections. This study was supported by National Research Foundation of Korea (grant no: NRF-2019R1C1C1002466) and Korea Polar Research Institute (grant no: PE21060). DS-F was supported by the Australian Research Council (FT180100216). 


\section{References}

Alla, S.K., Clark, J.F. \& Beyette, F.R. (2009). Signal processing system to extract serum bilirubin concentration from diffuse reflectance spectrum of human skin. In: 2009 Annual International Conference of the IEEE Engineering in Medicine and Biology Society. IEEE, pp. 1290-1293.

Arnold, J.W. (1964). Blood circulation in insect wings. Mem. Entomol. Soc. Canada, 96, 5-60.

Ashton, K.G. (2002). Patterns of within-species body size variation of birds: strong evidence for Bergmann's rule. Glob. Ecol. Biogeogr., 11, 505-523.

Benjamini, Y. \& Hochberg, Y. (1995). Controlling the false discovery rate: a practical and powerful approach to multiple testing. J. R. Stat. Soc. Ser. B , 57, 289-300.

van der Bijl, W., Zeuss, D., Chazot, N., Tunström, K., Wahlberg, N., Wiklund, C., et al. (2020). Butterfly dichromatism primarily evolved via Darwin's, not Wallace's, model. Evol. Lett. , 4, 545-555.

Bladon, A.J., Lewis, M., Bladon, E.K., Buckton, S.J., Corbett, S., Ewing, S.R., et al. (2020). How butterflies keep their cool: Physical and ecological traits influence thermoregulatory ability and population trends. $J$. Anim. Ecol. , 89, 2440-2450.

Bogert, C.M. (1949). Thermoregulation in reptiles, a factor in evolution. Evolution, 3, 195-211.

Burtt Jr, E.H. \& Ichida, J.M. (2004). Gloger's rule, feather-degrading bacteria, and color variation among song sparrows. Condor , 106, 681-686.

Chaplin, G. (2004). Geographic distribution of environmental factors influencing human skin coloration. Am. J. Phys. Anthropol. , 125, 292-302.

Cheng, W., Xing, S., Chen, Y., Lin, R., Bonebrake, T.C. \& NAKAMURA, A. (2018). Dark butterflies camouflaged from predation in dark tropical forest understories. Ecol. Entomol., 43, 304-309.

Chown, S.L. \& Gaston, K.J. (2010). Body size variation in insects: a macroecological perspective. Biol. Rev. , 85, 139-169.

Clavel, J., Escarguel, G. \& Merceron, G. (2015). mvMORPH: an R package for fitting multivariate evolutionary models to morphometric data.Methods Ecol. Evol. , 6, 1311-1319.

Clench, H.K. (1966). Behavioral thermoregulation in butterflies.Ecology , 47, 1021-1034.

Coffin, D. (2008). DCRAW: Decoding raw digital photos in linux.

Cuthill, I.C., Allen, W.L., Arbuckle, K., Caspers, B., Chaplin, G., Hauber, M.E., et al. (2017). The biology of color.Science, 357, eaan0221.

Delhey, K. (2017). Gloger's rule. Curr. Biol. , 27, R689-R691.

Delhey, K. (2018). Darker where cold and wet: Australian birds follow their own version of Gloger's rule. Ecography , 41, 673-683.

Delhey, K. (2019). A review of Gloger's rule, an ecogeographical rule of colour: definitions, interpretations and evidence. Biol. Rev. , 94, 1294-1316.

Delhey, K., Dale, J., Valcu, M. \& Kempenaers, B. (2019). Reconciling ecogeographical rules: rainfall and temperature predict global colour variation in the largest bird radiation. Ecol. Lett. , 22, 726-736.

Ducrest, A.-L., Keller, L. \& Roulin, A. (2008). Pleiotropy in the melanocortin system, coloration and behavioural syndromes. Trends Ecol. Evol. , 23, 502-510.

Fick, S.E. \& Hijmans, R.J. (2017). WorldClim 2: new 1-km spatial resolution climate surfaces for global land areas. Int. J. Climatol., 37, 4302-4315. 
Freckleton, R.P. (2009). The seven deadly sins of comparative analysis.J. Evol. Biol. , 22, 1367-1375.

Friedman, N.R. \& Remeš, V. (2017). Ecogeographical gradients in plumage coloration among Australasian songbird clades. Glob. Ecol. Biogeogr. , 26, 261-274.

Galván, I., Rodríguez-Martinez, S. \& Carrascal, L.M. (2018). Dark pigmentation limits thermal niche position in birds. Funct. Ecol. , 32, 1531-1540.

Gaston, K.J., Chown, S.L. \& Evans, K.L. (2008). Ecogeographical rules: elements of a synthesis. J. Biogeogr. , 35, 483-500.

Gilchrist, G.W. (1990). The Consequences of sexual dimorphism in body size for butterfly flight and thermoregulation. Funct. Ecol. , 4, 475-487.

Hegna, R.H., Nokelainen, O., Hegna, J.R. \& Mappes, J. (2013). To quiver or to shiver: increased melanization benefits thermoregulation, but reduces warning signal efficacy in the wood tiger moth. Proc. R. Soc. B Biol. Sci. , 280, 20122812.

Heinrich, B. (1974). Thermoregulation in endothermic insects.Science, 185, 747-756.

Hernandez, C.E., Rodriguez-Serrano, E., Avaria-Llautureo, J., Inostroza-Michael, O., Morales-Pallero, B., Boric-Bargetto, D., et al. (2013). Using phylogenetic information and the comparative method to evaluate hypotheses in macroecology. Methods Ecol. Evol. , 4, 401-415.

Kammer, A.E. \& Bracchi, J. (1973). Role of the wings in the absorption of radiant energy by a butterfly. Comp. Biochem. Physiol. Part A Physiol. , 45, 1057-1063.

Kapan, D.D. (2001). Three-butterfly system provides a field test of mullerian mimicry. Nature , 409, $338-340$.

Kingsolver, J.G. (1985). Thermal ecology of Pieris butterflies (Lepidoptera: Pieridae): a new mechanism of behavioral thermoregulation. Oecologia , 66, 540-545.

Kingsolver, J.G. (1987). Evolution and coadaptation of thermoregulatory behavior and wing pigmentation pattern in pierid butterflies. Evolution , 41, 472-490.

Kingsolver, J.G. (1988). Thermoregulation, flight, and the evolution of wing pattern in pierid butterflies: the topography of adaptive landscapes. Am. Zool. , 28, 899-912.

Konishi, S. \& Kitagawa, G. (1996). Generalised information criteria in model selection. Biometrika , 83, 875-890.

Lande, R. (1980). Sexual dimorphism, sexual selection, and adaptation in polygenic characters. Evolution, 292-305.

Medina, I., Newton, E., Kearney, M.R., Mulder, R.A., Porter, W.P. \& Stuart-Fox, D. (2018). Reflection of near-infrared light confers thermal protection in birds. Nat. Commun. , 9, 3610.

Munro, J.T., Medina, I., Walker, K., Moussalli, A., Kearney, M.R., Dyer, A.G., et al. (2019). Climate is a strong predictor of near-infrared reflectance but a poor predictor of colour in butterflies.Proc. R. Soc. B Biol. Sci. , 286, 20190234.

R Core Team. (2017). R A Language and Environment for Statistical Computing.

Rawlins, J.E. (1980). Thermoregulation by the black swallowtail butterfly, Papilio polyxenes (Lepidoptera: Papilionidae).Ecology , 61, 345-357.

Revell, L.J. (2012). phytools: An R package for phylogenetic comparative biology (and other things). Methods Ecol. Evol. , 3, 217-223. 
Ruxton, G.D., William, A.L., Sherratt, T.N. \& Speed, M.P. (2018).Avoiding attack: the evolutionary ecology of crypsis, warning signals, and mimicry . 2nd edn. Oxford University Press, New York.

Schweiger, O., Harpke, A., Wiemers, M. \& Settele, J. (2014). CLIMBER: Climatic niche characteristics of the butterflies in Europe.Zookeys, 65-84.

Shanks, K., Senthilarasu, S., ffrench-Constant, R.H. \& Mallick, T.K. (2015). White butterflies as solar photovoltaic concentrators.Sci. Rep., 5, 12267.

Silberglied, R.E. (1984). Visual communication and sexual selection among butterflies. In: The Biology of Butterflies (eds. Vane-Wright, R.I. \& Ackery, P.R.). Academic Press, pp. 207-223.

Stuart-Fox, D., Newton, E. \& Clusella-Trullas, S. (2017). Thermal consequences of colour and near-infrared reflectance. Philos. Trans. R. Soc. B Biol. Sci. , 372, 20160345.

Trullas, S.C., van Wyk, J.H. \& Spotila, J.R. (2007). Thermal melanism in ectotherms. J. Therm. Biol. , $32,235-245$.

Tsai, C.-C., Childers, R.A., Nan Shi, N., Ren, C., Pelaez, J.N., Bernard, G.D., et al. (2020). Physical and behavioral adaptations to prevent overheating of the living wings of butterflies. Nat. Commun. , 11, 551.

Wasserthal, L.T. (1983). Haemolymph flows in the wings of pierid butterflies visualized by vital staining (Insecta, Lepidoptera).Zoomorphology, 103, 177-192.

Wiemers, M., Chazot, N., Wheat, C.W., Schweiger, O. \& Wahlberg, N. (2020). A complete time-calibrated multi-gene phylogeny of the European butterflies. Zookeys , 938, 97.

Xing, S., Bonebrake, T.C., Tang, C.C., Pickett, E.J., Cheng, W., Greenspan, S.E., et al. (2016). Cool habitats support darker and bigger butterflies in Australian tropical forests. Ecol. Evol. , 6, 8062-8074.

Zink, R.M. \& Remsen Jr, J. V. (1986). Evolutionary processes and patterns of geographic variation in birds. Curr. Ornithol. , 4, 1-69.

Table 1. Significant predictors that explain the mean reflectance of each body region from PGLS analysis on the MCC tree after controlling for the false discovery rates. D: Dorsal, V: Ventral, B: Basal wings, E: Entire wings, T: Thorax.

\begin{tabular}{lllllll}
\hline Body region & DT & DB & DE & VT & VB & VE \\
\hline $\begin{array}{l}\text { Significant } \\
\text { terms for } \\
\text { overall }\end{array}$ & PC1 $(<0.001)$, & PC1 $(0.002)$, & PC1 $(0.006)$, & PC1 $(<0.001)$ & PC1 $(<0.001)$, & PC1 $(0.008)$, \\
$\begin{array}{l}\text { reflectance } \\
\text { models }\left(P_{a d j}\right)\end{array}$ & & PC2 $(0.003)$ & $\begin{array}{l}\text { PC2 }(0.002), \\
\text { Size }(0.005)\end{array}$ & & PC2 $(0.04)$ & PC2 $(0.02)$, \\
$\begin{array}{l}\text { Significant }(0.009) \\
\text { terms for }\end{array}$ & PC2 (0.002) & PC2 $(0.007)$ & Size $(<0.001)$ & PC1 $(<0.001)$ & PC1 $(<0.001)$, & Size $(<0.001)$ \\
$\begin{array}{l}\text { residual } \\
\text { models }\left(P_{a d j}\right)\end{array}$ & & & & & PC2 $(0.007)$ & \\
\hline
\end{tabular}

Figure legends

Figure 1. PCA loadings on climatic factors (left) and the relationship between climatic PC1 and butterfly reflectance mapped on the butterfly phylogeny (right). The dimensionality of the six reflectance variables (mean reflectance of dorsal/ventral thorax, basal wings, and entire wings across 320 - $1050 \mathrm{~nm}$ range) was reduced using PCA. Reflectance PC1 explained $68 \%$ of the variation and correlated strongly with all six reflectance variables $(r>0.71)$. Colours in the phylogenetic tree indicate climatic PC1, and colours in the heatmap show butterfly reflectance PC1. 
Figure 2. Average reflectance of European butterfly species for each body region. The colour of each grid $(50 \times 50 \mathrm{~km})$ represents the average reflectance (over $320-1050 \mathrm{~nm}$ ) of all butterfly species assembly found in each grid. The colour code of each map was assigned using the Jenks natural break classification method to maximise the variance between each colour class. Red indicates that the butterfly assemblage in the area has higher reflectance while blue indicates lower reflectance ( $N=343$ species).

Figure 3. The relationship between climatic PC1 and the mean reflectance of each body region of butterflies. The trend lines represent the prediction from the multivariate phylogenetic regression models after accounting for the phylogenetic relationships.

Figure 4. The relationship between climatic PC1 and residuals of the model where near-infrared reflectance $(670-1050 \mathrm{~nm})$ was linearly fitted by log form of visible reflectance $(320-680 \mathrm{~nm})$. Only ventral thorax and ventral basal wing regions showed significant trends. The trend lines represent the prediction from the PGLS models.

Figure 5. The relationship between climatic PC1 and ventral-dorsal reflectance differences in butterflies. The difference was calculated by subtracting dorsal reflectance from ventral reflectance. The trend lines represent the prediction from the multivariate phylogenetic regression models.
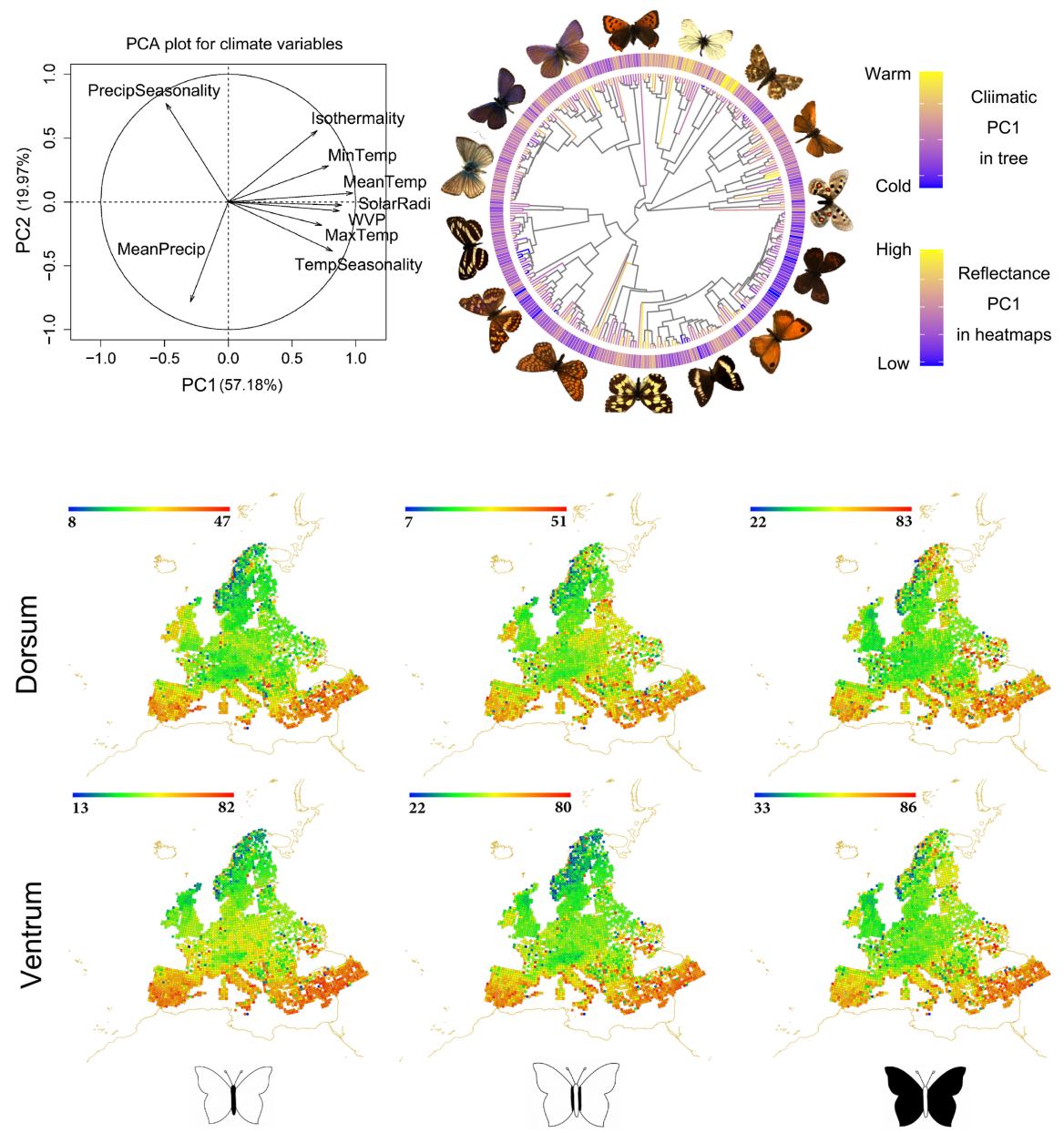

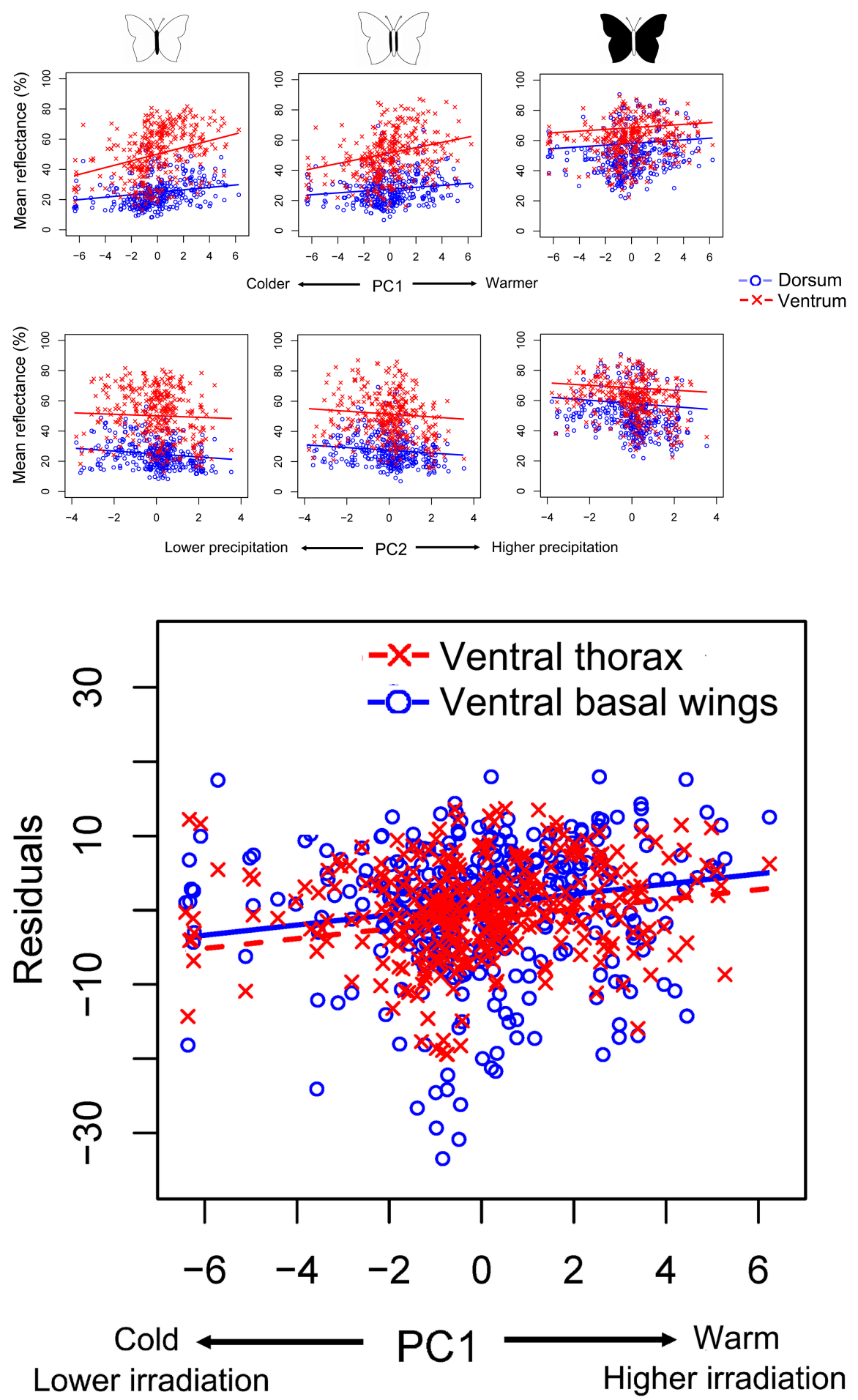

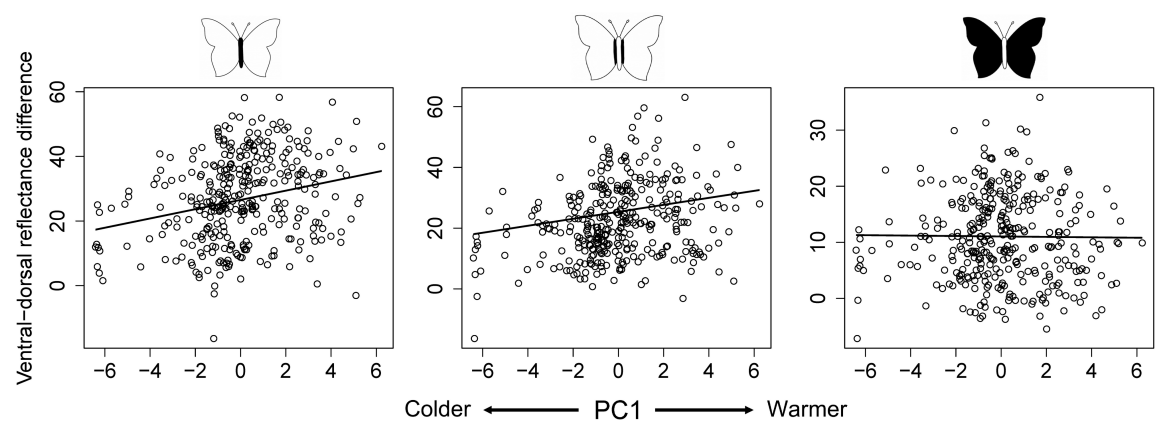